\title{
Caracterización de los programas académicos y determinación de las variables que afectan el rendimiento de los estudiantes en el Colegio de Estudios Superiores de Administración, CESA
}

\author{
Edgardo Cayón*, Juan Santiago Correa y Luz Elenny Scarpetta \\ Colegio de Estudios Superiores en Administración (CESA), Calle 35 No. 5 A-31, Bogotá-Colombia. \\ (correo-e: ecayon@cesa.edu.co; juansc@cesa.edu.co; luz.scarpetta@cesa.edu.co) \\ * Autor a quien debe ser dirigida la correspondencia
}

Recibido May. 6, 2021; Aceptado Jul. 7, 2021; Versión final Jun. 26, 2021, Publicado Oct. 2021

\begin{abstract}
Resumen
El presente estudio identifica las variables que tienen mayor influencia en el rendimiento académico y, por lo tanto, pueden ser predictores de la deserción estudiantil en el Colegio de Estudios Superiores de Administración (CESA, Bogotá, Colombia). Se utilizó la información de la Oficina Registro y Control que comprenden registros completos y sistematizados de 866 alumnos de pregrado y 189 de posgrado. Se usó tres modelos: uno de regresión simple, un modelo probit y un tercer modelo lineal con la información disponible de los posgrados. Los resultados mostraron que variables como el tipo de bachillerato de donde provenía el estudiante, su desempeño en la prueba nacional de ingreso y un buen desempeño en el proceso de admisión a la universidad incidieron positivamente en el desempeño de los estudiantes. Finalmente, los mejores predictores de la deserción estudiantil fueron la edad cuando los estudiantes ingresan a la Universidad y el género del estudiante.
\end{abstract}

Palabras clave: rendimiento académico, deserción estudiantil, educación superior, predicción de la deserción

\section{Characterization of academic programs and identification of variables that affect performance of students at the Higher Studies Business School, CESA}

\begin{abstract}
The aim of this paper is to identify the variables that have an impact on academic performance and therefore can predict academic desertion. For the study, a sample of 866 undergraduate and 189 graduate students from CESA Business School, located in Bogotá-Colombia, was used. Three models were used: a simple regression model, a probit model and a linear model using the information of the graduate programs. The aim was to determine which variables were statistically significant for determining academic performance and then tested the same variables as a predictor of student desertion in a logit regression context. The results showed that variables such as the type of high school where the student came from, his/her performance on the national entrance exam, and a good performance in the university admission process had positive impact on student performance. Finally, the best predictors for student desertion were the age of the student at time of admission and the gender of the student.
\end{abstract}

Keywords: academic performance, student desertion, higher education, desertion prediction 


\section{INTRODUCCION}

En la Conferencia sobre Educación Superior de la UNESCO celebrada en París - del 5 al 8 de julio de 2009- se planteó la existencia de una relación directa entre mayores niveles de educación y de investigación, y la erradicación de la pobreza, la creación de modelos de desarrollo sostenible y el progreso a través de acuerdos internacionales sobre las metas de desarrollo. En este sentido, la UNESCO afirmó: [...] como bien público e imperativo estratégico para todos los niveles de enseñanza (así como para las bases de la investigación, la innovación y la creatividad) alcanzar mayores niveles de educación debe ser un asunto de responsabilidad y apoyo económico para todos los gobiernos (UNESCO, 2009).

En las últimas dos décadas se ha registrado, a nivel mundial, una serie de esfuerzos para mejorar la calidad y el acceso a la educación superior; sin embargo, el acceso en sí mismo es insuficiente, en tanto debe existir una política para asegurar la permanencia y la culminación de los estudios de todas las personas que ingresan al sistema (UNESCO, 2009). En la sociedad actual, la formación terciaria y posgradual se ha convertido en una prioridad en la política social. Las corrientes de pensamiento contemporáneas consideran que los niveles de desigualdad socio-económica pueden ser medidos en términos de una mayor o menor educación recibida, razón por la cual la instrucción se ha convertido en un imperativo y en una obligación primordial de los Estados (Universidad Nacional, 2009).

Aunque la tendencia global revela una mejora del acceso a la educación superior en términos del aumento del ingreso de estudiantes al sistema, aún persisten importantes disparidades que se han convertido en una fuente significativa de desigualdad. Uno de los mayores problemas que genera la expansión del acceso a la educación superior radica en su calidad. La calidad se debe reflejar todos los objetivos de la educación superior y debe ser una meta a cultivar entre los estudiantes con pensamiento crítico e independiente a través de la capacidad de "aprender a lo largo de la vida" (UNESCO, 2009). En este contexto, la deserción estudiantil se convierte en un problema de equidad social que perpetúa situaciones de exclusión social y económica. En primer lugar, la deserción retroalimenta los círculos de pobreza y el potencial aumento del subempleo. En segundo lugar, limita las misiones institucionales en tanto disminuye los índices de eficiencia y calidad con claras implicaciones económicas para las universidades. Y, en tercer lugar, la deserción genera sentimientos de malestar y frustración en las personas que abandonan sus estudios, por las menores probabilidades de obtener empleos satisfactorios, así como la obtención de salarios bajos (González, 2006).

El acceso a la educación se debe articular con la permanencia de los estudiantes en los programas, de tal manera que los diferentes grupos poblacionales concluyan exitosamente su proceso de formación. En la actualidad es ineludible el desarrollo de nuevos sistemas de apoyo al estudiantado y aproximaciones innovadoras a la pedagogía; más aún en economías emergentes en las que existe una incongruencia entre la necesidad de educar especialistas y profesionales técnicos, por una parte, y, por otra, formar líderes con un conocimiento general que les permita ser creativos, adaptables y capaces de dar amplias consideraciones éticas de los avances sociales (Altbach, et al., 2009).

El comunicado de la Conferencia sobre Educación Superior (De la Fuente, 2009) hizo énfasis en que la enseñanza superior debe perseguir simultáneamente los "objetivos de equidad, pertinencia y calidad", y resaltó la importancia de reforzar los procesos de aseguramiento de la calidad, entre los cuales la disminución de los niveles de deserción en la educación superior en un indicador central en la medición de la eficiencia del sistema y de mejores niveles de acceso y de equidad en la educación, asegurando así el "atractivo de la carrera universitaria" (De la Fuente, 2009). Para abordar el caso concreto de la Institución de Educación Superior -IESque se analiza en este documento: el Colegio de Estudios Superiores de Administración -CESA-, vale mencionar que fue creado en 1974 por iniciativa de un grupo de figuras públicas y empresarios privados con el apoyo de la Asociación Nacional de Industriales -ANDI-, y del Instituto Colombiano de Administración INCOLDA-. Inició labores en 1975 con el objetivo de "formar los mejores líderes empresariales, dotados de espíritu emprendedor, capaces de agregar valor a sus empresas, creativos, éticos y comprometidos con la generación de desarrollo económico, político y social".

Desde sus inicios la Institución ha tenido un carácter monodisciplinar, tanto en su programa de pregrado en Administración de Empresas, como en sus programas de posgrados en la misma área disciplinar. Igualmente, el CESA ha estado orientado por la filosofía de educación personalizada. Y precisamente este modelo de educación comprende no sólo mantener grupos de estudiantes reducidos, sino que ha exigido el diseño de una serie de políticas y estrategias que aseguren el éxito en la culminación de sus estudiantes dentro de altos estándares de calidad en su formación. De esta manera se han logrado evitar las situaciones de deserción de estudiantes de la Institución.

El proceso de admisiones del CESA comprende dos requisitos: el resultado de las pruebas estatales (ICFES Saber 11) y entrevistas personalizadas. Estas son realizadas por un grupo de profesionales que evalúan la claridad del estudiante frente al programa; sus características personales con relación a los objetivos de 
formación del CESA, sus cualidades de liderazgo, emprendimiento y responsabilidad. De los dos instrumentos, el segundo tiene un mayor peso en la selección de los aspirantes porque permite seleccionar alumnos con altos niveles de identificación con el programa y con los objetivos de formación específicos del CESA. De manera que se reduce el riesgo de deserción en los primeros semestres de formación, situación que se suele presentar en las IES.

EI CESA realiza acompañamiento académico en los primeros cuatro semestres de formación profesional a aquellos estudiantes con dificultades en el desarrollo de habilidades específicas, concretamente de lengua a través del Centro de apoyo académico DIGA, y matemáticas por parte del Centro de apoyo académico SUMA. Adicionalmente, la Institución cuenta con un programa de identificación temprana de estudiantes con problemas personales o familiares que puedan implicar abandono del programa de estudios. Para esto, la Consejería Académica y Sicológica realiza seguimiento y acompañamiento a los estudiantes para disminuir los niveles de riesgo de deserción. Para casos especiales de enfermedad, hay programas que le permiten a los estudiantes un manejo más flexible de su carga académica semestral, lo mismo que del número de horas presenciales que requieren para aprobar los cursos en los que están matriculados. En cuanto a las razones económicas, a pesar de no ser la causa principal de deserción en el CESA, la Institución implementó un programa de apoyos financieros para casos específicos. Estos apoyos se dan en dos modalidades: rebajas en los costos de los derechos de matrículas o el pago diferido de estos valores.

Estos esquemas se han logrado en gran medida gracias al perfeccionamiento continuo de un modelo de educación personalizada en el que todas las fases del entorno del estudiante se analizan desde una perspectiva integral. El mapa conceptual en el que se basa el modelo de educación personalizada del CESA se revisa de manera permanente a la luz de los paradigmas que presentan los diferentes problemas en la implementación práctica de un sistema de gestión universitaria que se adecúa a un objetivo educativo común: la reducción de la deserción estudiantil.

No obstante, aunque los resultados generales son satisfactorios para el CESA, sería valioso tener una caracterización precisa y completa de la población estudiantil de pregrado y posgrado, además de contar con datos que permitan evidenciar de manera temprana situaciones que puedan convertirse en predictores de rendimiento académico, tiempo de permanencia, situación económica y éxito o fracaso académico. Tener bases de datos con edades, género, colegios y universidades de origen, estrato socioeconómico, trayectorias familiares en la Comunidad CESA, entre otras, que permitan estimar modelos para prever el potencial de éxito y fracaso de un estudiante en el CESA. Esta información permitiría una toma de decisiones más asertiva para potenciar las ventajas existentes, con miras a lograr egresados más competitivos en el mercado laboral y con un potencial de desarrollo profesional mayor. El objetivo del presente estudio es doble: en primer lugar, se pretende caracterizar la población estudiantil en cuanto a graduados y estudiantes activos del CESA, y en segundo lugar, se busca identificar los factores que tienen más influencia en el rendimiento académico y que, por ende, puedan servir de predictores de la deserción estudiantil.

\section{OTROS ANTECEDENTES}

La deserción estudiantil es uno de los principales problemas en las Instituciones de Educación Superior por el impacto que representa para la institución y para la sociedad el que un estudiante desista de su programa de estudios. La deserción universitaria se entiende como el abandono del programa de estudios antes de completar el grado académico, lo que se puede entender como una ineficiencia del sistema educativo tanto del estado como de la institución misma. Según la Organización para la Cooperación y el Desarrollo Económico -OCDE- se estima que el $30 \%$ de los estudiantes que ingresan a un programa universitario abandonan sus estudios. Gallegos et al. (2018) evidencian diferencias entre países, por ejemplo, en Australia, Dinamarca, Japón y Francia se tiene una tasa del 20\%, mientras que en Chile, Hungría, Suiza y Estados Unidos alcanzan niveles del $50 \%$.

A continuación, se describen algunas investigaciones realizadas en diferentes países que pretenden determinar los factores que más impactan el porcentaje de alumnos que se retiran de su plan de estudios. Para el caso de Chile, por ejemplo, Gallegos et al. (2018) analizaron una muestra de 1.565 estudiantes matriculados en el período 2004-2013 en la Universidad Católica de la Santísima Concepción de Chile, con el fin de analizar la deserción no sólo en el primer año de estudios sino también en las anualidades subsiguientes. En el análisis econométrico se incorporaron variables de carácter socio-demográficas, socioeconómicas, y académicas, para concluir que la región de procedencia del estudiante, el promedio ponderado y el acceso a becas o financiamiento afectan directamente la probabilidad de deserción de un estudiante, además el rendimiento académico es la variable más influyente, y su peso relativo aumenta a medida que el estudiante avanza en su proceso de formación. De otro lado, el nivel de ingresos o la educación de los padres no explican la deserción, mientras que el lugar de procedencia del estudiante afecta la probabilidad de deserción durante el primer año. Los resultados del modelo probabilístico arrojaron tasas de deserción de $36,41 \%$ de probabilidad al primer año, un $39,83 \%$ al segundo, y un $42,26 \%$ al tercer año. 
Para el caso específico de escuelas de negocios, la investigación de Mangum et al. (2005), identificó las variables más relevantes que pueden afectar la deserción de los estudiantes de este tipo de profesión universitaria. Para el estudio tomaron como base una muestra de 403 estudiantes de segundo semestre y analizaron los datos de deserción del mismo grupo de estudiantes cuatro semestres después de la primera fase. Como resultado se identificaron tres factores que representaron una correlación significativa con la deserción: promedio de calificaciones de primer semestre, promedio de evaluación del curso de primer semestre y la percepción de dificultades financieras. Otros estudios se han centrado en la identificación de acciones que pueden influir en la permanencia de los estudiantes; es el caso de la investigación realizada por Casanova et al. (2018), en la Universidad Pública del Norte de Portugal. En esta se sugiere la intervención de la institución para lograr altos índices de permanencia de sus alumnos en el primer año, no solo basados en el rendimiento académico sino también con el análisis de variables en los procesos de selección que permitan predecir el comportamiento académico de un estudiante y focalizar las estrategias para prevenir su deserción. De igual forma buscar los instrumentos de motivación adecuados para que un estudiante que inició un programa de estudios como segunda opción pueda adaptarse y continuar con este plan de estudios alterno.

La investigación realizada por Gitto et al. (2016), con datos de 76 universidades italianas, concluye que la localización de la institución educativa influye en la deserción y también factores de oferta como el número de campus remotos universitarios y el número de cursos impartidos en esas sedes. El tener un campus cerca a su lugar de residencia motiva a los estudiantes a inscribirse, adicionalmente tienen mayor motivación en obtener créditos en comparación con los estudiantes que se inscriben lejos de su lugar de residencia, una universidad menos dispersa podría ofrecer un ambiente académico más efectivo y, por ende, ayuda a reducir las tasas de deserción. Los mejores predictores para el promedio académico están determinados por la autoeficiencia académica y la motivación al logro (Robbins et al., 2004). En suma, la calidad del aprendizaje y la permanencia de los estudiantes en el programa dependen de una interacción entre los tipos de objetivos sociales y académicos que los estudiantes traen al aula, las propiedades motivadoras de estos objetivos y las estructuras de recompensa en el aula.

Para el caso de España, Lassibille y Navarro (2008) analizaron otras variables, como la edad de ingreso al programa académico y la preparación previa. Los resultados muestran un comportamiento de abandono particularmente alto. Al momento de la encuesta, alrededor del $40 \%$ de los estudiantes desertaron del programa académico. Los estudiantes que retrasan el ingreso a la educación superior, así como los estudiantes que no ingresaron al curso que tenían como primera opción, están en mayor riesgo de deserción. Los autores evidencian que en la universidad los estudiantes que ingresan con un diploma de educación vocacional no están suficientemente preparados y no tienen las habilidades necesarias para tener éxito en la educación superior. Otro hallazgo consiste en que los estudiantes que reciben apoyo financiero tienen tasas de deserción escolar más bajas que aquellos que no reciben apoyo y, por último, que el impacto del apoyo financiero es particularmente importante al inicio de la carrera escolar.

Para el caso de Londres, el estudio de Bennett (2003), realizado en la Universidad Metropolitana de Londres, muestra que las dificultades financieras tuvieron un alto impacto en la decisión de abandonar o permanecer en el programa académico mientras que se tiene un impacto moderado en las variables de rendimiento académico y el nivel de compromiso del estudiante con su programa. En Colombia, se tiene la investigación realizada por Cayón y Correa (2014), frente a la retención estudiantil en el Colegio de Estudios Superiores de Administración -CESA- en la cual se evidencia que el tener un proceso de selección riguroso y un monitoreo permanente del estudiante genera un impacto positivo en la retención del estudiante. Nuestra investigación se centra en las variables de deserción mencionadas en diversos casos de estudio sobre deserción universitaria como Quintero-Guasca et al. (2021) en el que resaltan que la permanencia estudiantil se basa en factores de tipo individual y socio económico, y en el caso de universidades privada colombianas como es el objeto de nuestro estudio, Castro-Montoya et al. (2021) demostraron que aparte de las variables socioeconómicas y académicas, las variables demográficas de sexo y edad tenían un impacto importante en la deserción universitaria.

\section{METODOLOGIA}

Para el presente estudio se hace un análisis cuantitativo para analizar cuáles son las variables que tienen más incidencia en la deserción del CESA. Para tal fin, primero se hizo un modelo de regresión simple para determinar cuáles son las variables estadísticamente significativas para predecir el promedio acumulado en el programa académico durante su estadía en el CESA. El modelo que se estimó se presenta en la ecuación (1).

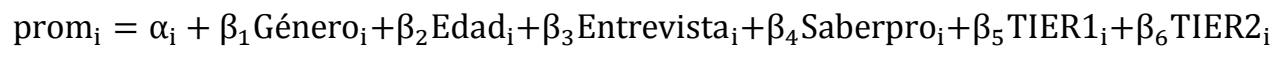

$+\beta_{7}$ TIER $_{\mathrm{i}}+\beta_{8}$ UNCOLI $_{\mathrm{i}}+\beta_{9}$ FamiliaCesa $_{\mathrm{i}}+\beta_{1}$, $_{\text {Elite }}+\varepsilon$ 
Donde: prom $_{i}=$ promedio acumulado del estudiante durante el programa de estudio, género $i=$ es una variable dummy que toma el valor de 1 si el sexo del estudiante es masculino y 0 si es femenino, edadi $=$ es la edad del estudiante en el momento que ingresa al CESA, entrevistai = es el puntaje obtenido por el estudiante en la entrevista personal del CESA, Saberproi = es el puntaje obtenido por el estudiante en el examen de estado, $\mathrm{TIER}_{1}$, TIER 2 , TIER 3 son variables dummies que toman el valor de 100 , si el colegio de donde proviene el estudiante de pregrado pertenece a uno de los grupos ahí identificados.

EI CESA categoriza los colegios de donde provienen los estudiantes de pregrado de acuerdo con una serie de características que contemplan el costo del colegio, las acreditaciones, localización geográfica, etc. Siendo TIER 1 los colegios que históricamente han estado más vinculados con el CESA y TIER 2 y 3 , son los colegios que no tienen una vinculación tan estrecha como el CESA pero que son importantes en el mediano y largo plazo para consolidar el programa de pregrado de la institución. Elite $e_{i}$ es una variable dummy que toma el valor de 1 si el estudiante tomó el programa preuniversitario del CESA antes de entrar o 0 si no lo tomó. Una vez se estimó el modelo y se revisaron las variables se procedió a estimar modelo probit de la ecuación (2) con las mismas variables. Nuestra hipótesis es que las variables aquí definidas son estadísticamente significativas en explicar el promedio académico de los estudiantes y por ende las causas de deserción en el CESA.

$\operatorname{Pr}\left(D_{i}=1 / X_{i}\right)=\left(1+\exp \left(-\left(\alpha_{i}+\beta_{1}\right.\right.\right.$ Género $_{i}+\beta_{2}$ Edad $_{i}+\beta_{3}$ Entrevista $_{i}$

$+\beta_{4}$ Saberpro $_{\mathrm{i}}+\beta_{5}$ TIER $_{\mathrm{i}}+\beta_{6}$ TIER $_{\mathrm{i}}+\beta_{7}$ TIER $_{\mathrm{i}}+\beta_{8}$ UNCOLI $_{\mathrm{i}}+\beta_{9}$ FamiliaCesa $_{\mathrm{i}}+\beta_{10}$ Elite $\left.\left._{\mathrm{i}}\right)\right)^{-1}$

Donde $D_{i}=$ es una variable dummy que toma el valor de 1 si el alumno desertó y 0 si se graduó o continua activo. El resto de las variables son las mismas de la ecuación (1). Finalmente, dado que la información de posgrado era muy limitada para poder hacer el modelo probit de deserción, utilizamos el modelo de la ecuación 3 para determinar cuáles variables tienen relación con el promedio académico del estudiante.

$\operatorname{prom}_{\mathrm{i}}=\alpha_{\mathrm{i}}+\beta_{1}$ Género $_{\mathrm{i}}+\beta_{2}$ Edad $_{\mathrm{i}}+\beta_{3}$ Instituciónacreditada $_{\mathrm{i}}+\varepsilon$

Donde género y edad se definen de la misma manera que la ecuación (1) e instituciónacreditadai es una variable dummy que toma el valor de 1 si el estudiante de posgrado viene de una institución acreditada institucionalmente y 0 si no lo es. A continuación, hacemos una descripción en detalle de la data que se empleo en los modelos propuestos anteriormente.

\section{Descripción de los datos}

Los datos utilizados para las variables independientes y dependientes expuestas en la metodología del presente estudio pertenecen al sistema de información de la Oficina de Registro y Control del CESA; que contenía registros de 13.704 alumnos de pregrado y posgrado. En la tabla 1 se presenta la composición de la población por género y programa. El pregrado se compone exclusivamente por el programa de administración de empresas, y el posgrado por los programas restantes.

Tabla 1. Caracterización por género de la población del CESA estudiantes graduados, activos e inactivos. Información de la Oficina de Registro y Control del CESA.

\begin{tabular}{|l|c|c|c|}
\hline & Total & Pregrado & Posgrado \\
\hline Femenino & 6.213 & 3.200 & 3.013 \\
\hline Masculino & 7.491 & 4.571 & 2.920 \\
\hline Total & 13.704 & 7.771 & 5.933 \\
\hline & $\begin{array}{c}\text { Administración de } \\
\text { empresas }\end{array}$ & $\begin{array}{c}\text { Especialización en finanzas } \\
\text { corporativas }\end{array}$ & $\begin{array}{c}\text { Especialización en mercadeo } \\
\text { estratégico }\end{array}$ \\
\hline Femenino & 3.200 & 663 & 1.646 \\
\hline Masculino & 4.571 & 954 & 2.176 \\
\hline Total & 7.771 & 1.617 & Maestría en Finanzas Corporativas \\
\hline & Maestría en & Maestría en Dirección de Marketing & 239 \\
\hline Femenino & 111 & 354 & 369 \\
\hline Masculino & 187 & 234 & 608 \\
\hline Total & 298 & 588 & \\
\hline
\end{tabular}


Históricamente, el CESA ha tenido una paridad de género en su población estudiantil, lo cual se refleja en la muestra analizada, donde las mujeres representan el $51 \%$ en posgrados, y el $45 \%$ en el pregrado. Los programas con menos igualdad de género son las Maestrías en Administración de Empresas y en Finanzas Corporativas, donde las mujeres representan el 37\% y 39\% respectivamente. Los alumnos que actualmente cursan, han cursado, o dejaron de cursar un programa de estudios en el CESA se clasifican en las siguientes categorías: Activo: alumno vinculado a un programa de estudios, Borrado financieramente: alumno que pasó proceso de admisión y no se matriculó (no hizo el pago del semestre), Cancelado: alumno que tiene matrícula académica y financiera pero que durante el semestre se retira por un semestre o indefinido con posibilidad de reintegro, Egresado: alumno que terminó su plan de estudios, Excluido: alumno que por condiciones académicas no puede volver a la Institución, Expulsado: alumno por condiciones disciplinarias no puede volver a la institución, Graduado: alumno que cumple requisitos de documentos y paz y salvos para recibir su título de grado, Inactivo: alumno que fue suspendido, tiene reserva de cupo o no realiza el pago para el siguiente semestre, Inscrito: persona aspirante que no fue admitida, y Traslado: alumno que cambia de un programa a otro o de un pensum a otro. En la tabla 2 presenta el estado académico de los alumnos por programa, y podemos observar que la cifra de alumnos cancelados e inactivos son aquellos que más pueden tener riesgo de deserción y son una parte importante de la población que curso o está cursando un programa de estudios en el CESA. Si consolidamos la información de la tabla 2 podemos observar que a la fecha de este estudio esta cifra se ubica históricamente alrededor del $18,33 \%$ de la población total.

Tabla 2. Estado académico de población del CESA por programa de estudio. Información de la Oficina de Registro y Control del CESA.

\begin{tabular}{|l|r|r|}
\hline Administración de empresas & 7.771 & Porcentaje (\%) \\
\hline Activo & 1.111 & 14,30 \\
\hline Borrado financieramente & 555 & 7,10 \\
\hline Cancelado & 31 & 0,40 \\
\hline Egresado & 110 & 1,40 \\
\hline Excluido & 212 & 2,70 \\
\hline Expulsado & 4 & 0,10 \\
\hline Graduado & 3.665 & 47,20 \\
\hline Inactivo & 2.069 & 26,60 \\
\hline Inscrito & 2 & 0,00 \\
\hline Traslado & 12 & 0,20 \\
\hline Especialización en Finanzas Corporativas & 1.617 & \\
\hline Activo & 91 & 5,60 \\
\hline Borrado financieramente & 48 & 3,00 \\
\hline Cancelado & 1 & 0,10 \\
\hline Egresado & 17 & 1,10 \\
\hline Excluido & 4 & 0,20 \\
\hline Graduado & 1.335 & 82,60 \\
\hline Inactivo & 121 & 7,50 \\
\hline Especialización en Mercadeo Estratégico & 2.822 & \\
\hline Activo & 120 & 4,30 \\
\hline Borrado financieramente & 101 & 3,60 \\
\hline Cancelado & 2 & 0,10 \\
\hline Egresado & 30 & 1,10 \\
\hline Excluido & 5 & 0,20 \\
\hline Graduado & 2.365 & 83,80 \\
\hline Inactivo & 199 & 7,10 \\
\hline Maestría en Administración & 297 & \\
\hline Activo & 118 & 39,73 \\
\hline Borrado financieramente & 50 & 16,84 \\
\hline Cancelado & 8 & 2,69 \\
\hline Egresado & 1 & 0,34 \\
\hline Excluido & 5 & 1,68 \\
\hline Graduado & 100 & 33,67 \\
\hline Inactivo & & 5,05 \\
\hline & \\
\hline & 15 & \\
\hline
\end{tabular}


Tabla 2: continuación

\begin{tabular}{|l|c|c|}
\hline Maestría en Dirección de Marketing & 588 & Porcentaje (\%) \\
\hline Activo & 128 & 21,80 \\
\hline Borrado financieramente & 51 & 8,70 \\
\hline Cancelado & 7 & 1,20 \\
\hline Egresado & 21 & 3,60 \\
\hline Excluido & 5 & 0,90 \\
\hline Graduado & 347 & 59,00 \\
\hline Inactivo & 28 & 4,80 \\
\hline Traslado & 1 & 0,20 \\
\hline Maestría en Finanzas Corporativas & 608 & \\
\hline Activo & 123 & 20,20 \\
\hline Borrado financieramente & 46 & 7,60 \\
\hline Cancelado & 4 & 0,70 \\
\hline Egresado & 24 & 3,90 \\
\hline Excluido & 7 & 1,20 \\
\hline Expulsado & 3 & 0,50 \\
\hline Graduado & 373 & 61,30 \\
\hline Inactivo & 27 & 4,40 \\
\hline Inscrito & 1 & 0,20 \\
\hline
\end{tabular}

Cuando se categoriza los datos por programa, se encuentra la mayor deserción o riesgo de deserción está en el pregrado con un 27\%, la Maestría en Administración de Empresas con un 7,7\%, la Especialización en Finanzas Corporativas con un 7,5\%, la Especialización en Mercadeo Estratégico con un 7,1\%, la Maestría en Dirección en Marketing con un 6,0\% y la Maestría en Finanzas Corporativas con un 5,1\%. Para tener otra perspectiva de análisis se construyó la tabla 3 con la composición de los programas académicos por grupos etarios.

Tabla 3. Rango de edades de la población del CESA en pregrado y posgrado. Datos de la Oficina de Registro y Control del CESA.

\begin{tabular}{|c|c|c|}
\hline & Cantidad & Porcentaje (\%) \\
\hline Administración de empresas & 7.771 & \\
\hline$<=18$ años & 4.980 & 64,1 \\
\hline entre 19 y 25 años & 2.636 & 33,9 \\
\hline entre 26 y 30 años & 46 & 0,6 \\
\hline entre 31 y 35 años & 16 & 0,2 \\
\hline$>36$ años & 93 & 1,2 \\
\hline Especialización en Finanzas Corporativas & 1.617 & \\
\hline$<=18$ años & 2 & 0,1 \\
\hline entre 19 y 25 años & 419 & 25,9 \\
\hline entre 26 y 30 años & 788 & 48,7 \\
\hline entre 31 y 35 años & 270 & 16,7 \\
\hline$>36$ años & 138 & 8,5 \\
\hline Especialización en Mercadeo Estratégico & 2.822 & \\
\hline$<=18$ años & 18 & 0,6 \\
\hline entre 19 y 25 años & 646 & 22,9 \\
\hline entre 26 y 30 años & 1.590 & 56,3 \\
\hline entre 31 y 35 años & 401 & 14,2 \\
\hline$>36$ años & 167 & 5,9 \\
\hline
\end{tabular}


Tabla 3: continuación

\begin{tabular}{|l|c|c|}
\hline & Cantidad & Porcentaje (\%) \\
\hline Maestría en Administración & 297 & \\
\hline$<=18$ años & 0 & 0,0 \\
\hline entre 19 y 25 años & 18 & 6,1 \\
\hline entre 26 y 30 años & 129 & 43,4 \\
\hline entre 31 y 35 años & 95 & 32,0 \\
\hline$>36$ años & 55 & 18,5 \\
\hline Maestría en Dirección de Marketing & 588 & \\
\hline$<=18$ años & 1 & 0,1 \\
\hline entre 19 y 25 años & 42 & 7,14 \\
\hline entre 26 y 30 años & 358 & 60,8 \\
\hline entre 31 y 35 años & 137 & 23,9 \\
\hline$>36$ años & 50 & 8,5 \\
\hline Maestría en Finanzas Corporativas & 608 & \\
\hline$<=18$ años & 3 & 0,5 \\
\hline entre 19 y 25 años & 158 & 26,0 \\
\hline entre 26 y 30 años & 282 & 46,4 \\
\hline entre 31 y 35 años & 96 & 15,8 \\
\hline$>36$ años & 69 & 11,3 \\
\hline
\end{tabular}

En posgrados de acuerdo a la información contenida en la tabla 3 , se puede observar que el promedio de edad de los estudiantes se encuentra entre los 26 y 30 años, mientras que en la Maestría en Administración de Empresas más del $50 \%$ de ellos tienen 30 años o más años. En el pregrado, históricamente, más del $60 \%$ de los estudiantes son menores de 18 años al momento del ingreso a la Institución educativa. Cuando se analiza la data histórica de cada programa se puede apreciar que en el sistema se encuentra una pequeña parte de la información disponible en estado físico. Por ejemplo, de los 7.771 alumnos de pregrado solo se cuenta con información completa sobre el puntaje del Saber Pro, colegios, tipo de colegio, edad de ingreso y puntaje de entrevista personal para 866 de ellos que es la data que se utilizó en el presente estudio.

En el caso de posgrado la situación es aún más compleja ya que de 5.993 estudiantes solo contamos con información para 189 de ellos y no se encuentran elementos importantes para el análisis en el nivel posgrado. Esto ocurre porque antes del año 2015 al sistema no se ingresaban las variables de ingreso al sistema. Lo mismo ocurre en posgrado que no se ingresa la información del empleo al momento de ingreso y/o el título de pregrado obtenido antes de ingresar al CESA. Y la buena parte de los campos con que cuenta el sistema para recolectar la información están vacíos, además de existir errores de tipografía (ejemplo un número muy bajo de estudiantes que tiene menos de 18 años, que se puede interpretar como alumnos de pregrado haciendo coterminales); esto para decir que el sistema está subutilizado y su contenido no permite hacer más categorizaciones que las expuestas en las tablas anteriores que se conviertan en información útil para la gestión y planeación académica.

\section{RESULTADOS}

En la tabla 4 se presentan los resultados obtenidos de la estimación de los modelos de las ecuaciones (1) a (3) expuestas en la sección de metodología, para determinar las variables que inciden en el promedio académico de pregrado, posgrado y la deserción en pregrado. De acuerdo con los resultados presentados en la tabla 4, todas las variables bajo estudio en pregrado son estadísticamente significativas con relación al promedio obtenido en el programa académico de pregrado. Las variables que mayor impacto positivo tienen en el promedio académico de acuerdo al coeficiente son: todas las relacionadas con el colegio de procedencia, especialmente los colegios clasificados TIER3i y si el estudiante curso el programa Elitei y las de relación inversa de acuerdo al coeficiente es la edad del estudiante y si el estudiante es hombre, estas variables explican en un $20 \%$ (R2) la variación del promedio académico, el restante puede ser atribuido a otras causas no observables. En el caso de posgrado la única variable que incide significativamente en el promedio obtenido es si el estudiante cursó su pregrado en una institución acreditada y su R2 es muy bajo para tener una conclusión definitiva. En el caso del modelo probit de deserción, las únicas variables que no son significativas con respecto a la deserción son la entrevista personal o si el estudiante pertenece a la familia CESA. 
Tabla 4. Resultados de los modelos estimados Nota: * ${ }^{* *} \mathrm{y}{ }^{* * *}$ indican el 90,95, y $99 \%$ de nivel de significancia respectivamente.

\begin{tabular}{|c|c|c|c|}
\hline & $\begin{array}{c}\text { Promedio } \\
\text { Carrera/Pregrado }\end{array}$ & $\begin{array}{l}\text { Deserción } \\
\text { / pregrado }\end{array}$ & $\begin{array}{c}\text { Promedio } \\
\text { Carrera/Posgrado }\end{array}$ \\
\hline \multirow[t]{2}{*}{ Género } & $-0,5165^{\star * *}$ & $0,4736^{\star * *}$ & 0,0544 \\
\hline & $(0,0759)$ & $(0,1258)$ & $(0,0879)$ \\
\hline \multirow[t]{2}{*}{ Edadi $_{i}$} & $-0,1889^{\star * *}$ & $0,1453^{\star \star}$ & $-0,0081$ \\
\hline & $(0,0438)$ & $(0,0648)$ & $(0,0099)$ \\
\hline \multirow[t]{2}{*}{ Entrevista $_{i}$} & $0,0051^{*}$ & $-0,0053$ & \\
\hline & $(0,0029)$ & $(0,0049)$ & \\
\hline \multirow[t]{2}{*}{ Saber Proi } & $0,0056^{\star * *}$ & $-0,0033^{\star *}$ & \\
\hline & $(0,0009)$ & $(0,0014)$ & \\
\hline \multirow[t]{2}{*}{ TIER1 $1_{i}$} & $0,7742^{* \star *}$ & $-1,1881^{* * *}$ & \\
\hline & $(0,1414)$ & $(0,1988)$ & \\
\hline \multirow[t]{2}{*}{ TIER2 } & $0,7166^{\star * *}$ & $-1,0339^{\star * *}$ & \\
\hline & $(0,1717)$ & $(0,2434)$ & \\
\hline \multirow[t]{2}{*}{ TIER3 $_{i}$} & $0,9092^{* * *}$ & $-1,3017^{* * *}$ & \\
\hline & $(0,1690)$ & $(0,2373)$ & \\
\hline \multirow[t]{2}{*}{ UNCOLI $_{\mathrm{t}}$} & $0,5153^{* * *}$ & $-0,8314^{* * *}$ & \\
\hline & $(0,0853)$ & $(0,1400)$ & \\
\hline \multirow[t]{2}{*}{ FamiliaCesa $_{i}$} & $0,1386^{*}$ & $-0,1624$ & \\
\hline & $(0,0822)$ & $(0,1293)$ & \\
\hline \multirow[t]{2}{*}{ Elite $_{i}$} & $0,7450^{\star * *}$ & $-1,0765^{\star * *}$ & \\
\hline & $(0,1363)$ & $(0,2967)$ & \\
\hline \multirow[t]{2}{*}{ Institución acreditada } & & & $0,1960^{* *}$ \\
\hline & & & $(0,0984)$ \\
\hline Adjusted $\mathrm{R}^{2} / \mathrm{McFadden}^{2}$ & 0,2061 & 0,1580 & 0,0295 \\
\hline Number of observations & 866 & 866 & 189 \\
\hline
\end{tabular}

En el caso de pregrado, y como lo habíamos mencionado anteriormente, los coeficientes betas de género y edad son significativos y con signo negativo, lo que significa que en el caso de los hombres su promedio en el programa académico es más bajo que el de las mujeres, y que los estudiantes con más edad al momento de ingreso, también tienden a tener un promedio general más bajo que el resto de la muestra. En el caso de la entrevista, el hecho que el estudiante sea familia CESA, y el Saber Pro, los coeficientes son positivos pero cercanos a cero, esto quiere decir que las variables a pesar de tener influencia en el promedio general de la carrera tienen menos impacto en el promedio general del estudiante que otras variables explicativas del modelo. En el caso de los otros coeficientes beta, los de mayor impacto en orden de relevancia son: si el alumno pertenece a un colegio TIER 3, TIER 1, atendió el programa Eli+e, pertenece a un colegio TIER 2 o a un colegio clasificado UNCOLI. Finalmente, en el caso de la deserción las variables que influyen positivamente en la probabilidad de deserción son el género (si el estudiante es hombre) y la edad (a mayor edad de ingreso por parte del estudiante, es más alta la probabilidad de deserción). Por el contrario, si el colegio es clasificado TIER 3, TIER 2, TIER 1, atendió el programa Eli+e o el colegio es clasificado UNCOLI, y el estudiante pertenece a alguno de estos grupos, la probabilidad de deserción (por causa de los coeficientes negativos) disminuye con respecto al resto de la población.

\section{DISCUSIÓN}

El análisis de los resultados de los modelos estimados permite identificar las variables que tienen más influencia en el rendimiento académico de los estudiantes del CESA, y que por ende sirven como predictores de la deserción estudiantil. En primer lugar, se encontró que el tipo de colegio de donde proviene el estudiante antes de ingresar al CESA tiene un efecto positivo en los resultados del estudiante en su programa, en especial para los estudiantes provenientes de colegios UNCOLI, colegios con una huella o tradición fuerte en el CESA y estudiantes provenientes de colegios por fuera de Bogotá que tienen un perfil similar a los de una tradición fuerte. En segundo lugar, se observa un impacto positivo y significativo de los estudiantes que cursaron previamente el programa Eli+e, con lo cual se valida el propósito de este al reforzar competencias 
necesarias para enfrentar la vida universitaria. En tercer lugar, existe una relación positiva y significativa de los estudiantes que tuvieron un puntaje alto en la prueba del Estado y un resultado alto en la entrevista; en particular este resultado es muy importante al validar las características fundamentales del proceso de admisión al pregrado de Administración de Empresas.

Un hallazgo importante que se evidenció fue que, para el pregrado en Administración de Empresas, las variables de género y edad son significativas y con signo negativo, es decir, que los hombres tienen un rendimiento académico más bajo que el de las mujeres, y que los estudiantes con más edad al momento de ingreso, también tienen a tener un promedio general más bajo que el resto de la muestra. Por otra parte, una de las variables que se tiene en cuenta en el proceso de selección, en la entrevista, es la de otorgar un puntaje adicional al aspirante que es hijo o familiar de un egresado del CESA. Lo que se evidenció en los resultados es que los coeficientes son positivos pero cercanos a cero, es decir, no es una variable significativa 0 importante para explicar el rendimiento académico de los estudiantes. Por último, en el caso de la deserción las variables que influyen positivamente en la probabilidad de deserción son el género (si el estudiante es hombre) y la edad (a mayor edad de ingreso por parte del estudiante, más alta es la probabilidad de deserción). Por el contrario, colegios con huella alta en el CESA o colegios UNCOLI, o si cursó el programa Eli+e, la probabilidad de deserción es baja respecto al resto de la población. Por su parte, ser hijo o familiar de egresados no tiene influencia en la deserción universitaria.

Con respecto a los programas de posgrados, a pesar de la escasez de datos en los sistemas de información, el modelo estimado permite inferir que los estudiantes que provienen de instituciones acreditadas tienen un promedio académico más alto que los egresados de instituciones no acreditadas. Cabe anotar que, en el presente estudio, se detectó que el sistema de información en el cuál basamos nuestro análisis se encuentra subutilizado, puesto que, a pesar de contar con información detallada de los estudiantes en medio físico, solo una pequeña parte de esta información se encuentra registrada correctamente en el sistema, lo que limita la capacidad de hacer análisis detallado sobre los determinantes del desempeño académico y por ende las causas de deserción. Una última consideración, tiene que ver con la disponibilidad de la información de la información de la Oficina Registro y Control del CESA. De los 7.771 registros impresos de alumnos de pregrado solo se cuenta con información completa en 866 casos. Y en el posgrado la situación es más aguda, pues de 5.993 estudiantes sólo se cuenta con información completa para 189 casos. En suma, el sistema ha sido subutilizado pues no se diligencian todos los campos de la información. Esta podría ser una recomendación para la Institución, y más allá una invitación a replicar este ejercicio con una muestra de información más amplia.

\section{CONCLUSIONES}

De acuerdo al trabajo presentado y a los resultados obtenidos, se pueden plantear las siguientes conclusiones principales:

1. Que las variables de género y edad son estadísticamente significativas al explicar la deserción. Es decir que los hombres y los estudiantes de mayor edad al momento de ingreso presentan un promedio académico más bajo y por ende una mayor probabilidad de deserción.

2. La variable que categoriza al colegio en el que el estudiante curso sus estudios de bachillerato es significativa y está relacionada positivamente con la reducción de la deserción ya que estos estudiantes cuentan con un mejor promedio y una tasa menor de deserción.

3. En el caso de posgrados los estudiantes procedentes de universidades que cuentan con alta acreditación de calidad tienen un promedio más alto que el de la muestra.

\section{REFERENCIAS}

Altbach, P. G., Reisberg, L., y Rumbley, L., Trends in Global Higher Education: Tracking an Academic Revolution, A report prepared for the UNESCO 2009 World Conference in Higher Education, UNESCO-SIDA/SAREC, Paris, Francia (2009)

Bennett, R., Determinants of Undergraduate Student Dropout Rates in a University Business Studies Department, https://doi.org/10.1080/030987703200065154, Journal of Further and Higher Education, 27(2), 123-141 (2003)

Casanova, J. R., Cervero, A., y otros tres autores, Factors that Determine the Persistence and Dropout of University Student, https://doi.org/10.7334/psicothema2018.155, Psicothema, 30(4), 408-414 (2018)

Castro-Montoya, B. A., Lopera-Gómez, y otros dos autores, Modelo de Riesgos Competitivos para Deserción y Graduación en Estudiantes Universitarios de Programas de Pregrado de una Universidad Privada de Medellín (Colombia), http://dx.doi.org/10.4067/S0718-50062021000100081, Form. Univ., 14(1), 81-98 (2021).

Cayon, E., y Correa, J. S., Personalized Education and its Role in Reducing Dropout Student Rates in Colombia: The CESA Case, European Journal of Social Science, 42(2), 255-263 (2014) 
De la Fuente, J. R., New Dynamics of Higher Education and Research for Societal Change and Development, Universidades, (42), 3-5 (2009)

Gallegos, J., Campos, N., y otros dos autores, Factores Determinantes en la Deserción Universitaria. Caso Facultad de Ciencias Económicas y Administrativas de la Universidad Católica de la Santísima Concepción, http://dx.doi.org/10.4067/S0718-50062018000300011, Form. Univ., 11(3), 11-18 (2018)

Gitto, L., Minervini., L. F., y Monaco, L., University Dropouts in Italy: Are Supply Side Characteristics Part of the Problem? https://doi.org/10.1016/j.eap.2015.12.004, Economic Analysis and Policy, 49, 108-116 (2016)

González, L. E., Repitencia y Deserción Universitaria, Informe sobre la Educación Superior en América Latina y el Caribe, 2000-2005: la metamorfosis de la educación superior por IESALC, 156-171, Editorial Metrópolis C.A., Caracas, Venezuela (2006)

Lassibille, G., y Navarro Gómez, L., Why do Higher Education Students Drop Out? Evidence from Spain. Education Economics, https://doi.org/10.1080/09645290701523267, 16(1), 89-105 (2008)

Mangum, W. M., Baugher, D., Winch, J. K., y Varanelli, A., Longitudinal Study of Student Dropout from a Business School, https://doi.org/10.3200/JOEB.80.4.218-221, Journal of Education for Business, 80(4), 218-221 (2005)

Quintero-Guasca, R. E., Avellaneda-Nieves, M., y otros dos autores, Permanencia Estudiantil en Programas de Posgrado E-learning: Un caso de Estudio, http://dx.doi.org/10.4067/S0718-50062021000300017, Form. Univ., 14(3), 17-24 (2021)

Robbins, S., Lauver, K., y otros tres autores., Do Psychosocial and Study Skill Factors Predict College Outcomes? A Metaanalysis, https://doi.org/10.1037/0033-2909.130.2.261, Psychological Bulletin, 130(2), 261-288 (2004) 
